\title{
Improved visual cognition through stroboscopic training
}

\author{
L. Gregory Appelbaum ${ }^{1,2}$, Julia E. Schroeder ${ }^{1,3}$, Matthew S. Cain ${ }^{1,3}$ and Stephen R. Mitroff ${ }^{1,3}$ * \\ Center for Cognitive Neuroscience, Duke University, Durham, NC, USA \\ ${ }^{2}$ Department of Psychiatry, Duke University, Durham, NC, USA \\ ${ }^{3}$ Department of Psychology and Neuroscience, Duke University, Durham, NC, USA
}

Edited by:

Peter J. Bex, Massachusetts Eye and Ear Infirmary, USA

Reviewed by:

Thomas S. A. Wallis, Schepens Eye

Research Institute, USA

Zhong-Lin Lu, University of Southern

California, USA

*Correspondence:

Stephen R. Mitroff, Center for

Cognitive Neuroscience, Duke

University, Box 90999, Durham, NC

27708, USA.

e-mail: mitroff@duke.edu
Humans have a remarkable capacity to learn and adapt, but surprisingly little research has demonstrated generalized learning in which new skills and strategies can be used flexibly across a range of tasks and contexts. In the present work we examined whether generalized learning could result from visual-motor training under stroboscopic visual conditions. Individuals were assigned to either an experimental condition that trained with stroboscopic eyewear or to a control condition that underwent identical training with non-stroboscopic eyewear. The training consisted of multiple sessions of athletic activities during which participants performed simple drills such as throwing and catching. To determine if training led to generalized benefits, we used computerized measures to assess perceptual and cognitive abilities on a variety of tasks before and after training. Computer-based assessments included measures of visual sensitivity (central and peripheral motion coherence thresholds), transient spatial attention (a useful field of view - dual task paradigm), and sustained attention (multiple-object tracking). Results revealed that stroboscopic training led to significantly greater re-test improvement in central visual field motion sensitivity and transient attention abilities. No training benefits were observed for peripheral motion sensitivity or peripheral transient attention abilities, nor were benefits seen for sustained attention during multiple-object tracking. These findings suggest that stroboscopic training can effectively improve some, but not all aspects of visual perception and attention.

Keywords: generalized learning, stroboscopic training, visual cognition, visual-motor control, plasticity

\section{INTRODUCTION}

A fundamental question about the nature of learning is how practice and prior experiences change an organism. The field of perceptual learning has long explored such issues with a specific focus on perception, and has typically found learning that does not broadly generalize. Human psychophysics research (e.g., Fahle and Poggio, 2002; Li et al., 2004) and computational models (e.g., Dosher and Lu, 1999) have revealed that prolonged practice with detecting or discriminating visual stimuli results in greater sensitivity to those stimuli, but that these benefits are highly specific. For example, training on discrimination of horizontal stimuli does not improve sensitivity to vertically oriented stimuli (Crist et al., 1997). Learning, therefore, appears to occur for the trained stimuli, with only a limited amount of transfer to other untrained tasks or stimuli (Goldstone, 1998; Ahissar and Hochstein, 2004). In recent years however, there have been a number of reports of generalized learning in which practice on specific tasks can result in beneficial transfer of learned abilities to other untrained domains (reviewed in Green and Bavelier, 2008). For example, the effects of musical training have been shown to improve spatiotemporal reasoning skills (Rauscher et al., 1997), verbal memory (Ho et al., 2003), and even general intelligence (Schellenberg, 2004). Similarly, extensive athletic training has been shown to improve performance on a number of measures of perception and cognition (reviewed in Mann et al., 2007), including covert attentional orienting (Lum et al., 2002), visual search and anticipation (Savelsbergh et al., 2002), motion speed and direction discrimination (Kioumourtzoglou et al., 1998), and response inhibition (Kida et al., 2005; but see Memmert et al., 2009).

In addition to the above examples ${ }^{1}$, a rapidly growing body of research has reported a strong link between experience with action video games and enhanced behavioral performance on a wide variety of perceptual and attention tasks (Green and Bavelier, 2003; Ferguson, 2007; Green et al., 2009) While this is still a developing enterprise, with much left to be determined (Boot et al., 2011), empirical demonstrations have shown that, among other cognitive benefits, avid action video game players possess increased visual sensitivity (Li et al., 2009), improved allocation of attention resources (Green and Bavelier, 2006a; Feng et al., 2007; Donohue et al., 2010), and more precise smooth pursuit eye movements (Tsoi et al., 2011). Recent computational models (Green et al., 2010) and theoretical reviews (Green et al., 2009) have led to the proposal that video game players are better able to form perceptual templates and derive probabilistic inference, suggesting that such generalized learning occurs where information is integrated and actions are selected.

Based upon the emerging video game training literature, there is a suggestion that activities that hold qualitative features that

\footnotetext{
${ }^{1}$ See also speech perception (Kraljic and Samuel, 2006), cognitive training (Mahncke et al., 2006; Smith et al., 2009), and driving (Crundall et al., 1999; Tsotsos et al., 2010).
} 
engage a high degree of perceptual or cognitive load in the service of accurate performance may lead to generalized learning effects. In the present research we explore this capacity for generalized learning by measuring perceptual and cognitive abilities that may be enhanced as a result of visual-motor training under stroboscopic visual conditions.

Fast-paced activities, such as those involved in competitive sports, place great demands on human vision. Actions and reactions are dependent on a constant supply of accurate and reliable information from the environment and, therefore, a premium is placed on rapid, distributed, and precise visual perception and attention abilities. One key aspect is the role of visual feedback it is often critical to be able to assess and update the relative movements, distances, and masses of objects in the visual environment in order to anticipate the appropriate forces required for a successful motor plan (Desmurget and Grafton, 2000). Motor actions are guided by a combination of central planning and online control provided in the form of feedback from the visual system about the moment-to-moment state of the world. Extensive research investigating the role of feedback on visual-motor control has demonstrated that movements become progressively more dependent on visual feedback with greater amounts of practice under conditions in which visual feedback is available (Proteau and Cournoyer, 1990; Proteau et al., 1992). Given this, we ask what might the implications be if visual feedback was interrupted, and individuals did not have access to this important control signal.

A new sports training tool (see Materials and Methods, below) has been designed based upon the premise that stroboscopic interruption of vision might enhance visual-motor control. The logic is that the interruption of visual information may force individuals to reduce their reliance on online visual feedback. It is thought that by practicing under situations of impoverished visual input, individuals will be forced to make better use of the limited visual information that is available. This, in turn, may train perceptual and attentional abilities that support basic visual-motor control. Previous research has indicated that stroboscopic training does, indeed, have benefits toward improved visual-motor actions such as driving performance (Tsimhoni et al., 1999), as well as reductions in symptoms that result from visual-motor disagreement, such as motion sickness (Reschke et al., 2006, 2007). Despite these premises and promising findings of specific visual-motor learning, it has not yet been explored whether stroboscopic training could result in generalized learning effects.

The goal of the present research was to begin to assess for improvements in visual cognitive abilities following stroboscopic training. For this purpose we used the recently developed Nike Vapor Strobe ${ }^{\circledR}$ eyewear. This eyewear uses battery-powered liquid crystal filtered lenses that alternate between clear and opaque states and provides varying lengths of occlusion that are under the users' control. The strobe effect is defined by opaque states that can vary through eight levels $(67-900 \mathrm{~ms})$, while the transparent state is fixed at a constant $100 \mathrm{~ms}(1-6 \mathrm{~Hz})$.

In theory, stroboscopic exposure may influence any of a number of perceptual or cognitive abilities. As such, we took an exploratory approach in order to assess for generalized transfer cognitive abilities due to stroboscopic training. We devised a series of computer-based and physical assessments to measure abilities before and after training. Participants either trained while wearing the Strobe eyewear or while wearing Control eyewear, an identical product except that it contained transparent lenses. For each assessment, the critical question was whether individuals who trained under stroboscopic conditions would improve significantly more from the pre-training assessments to the post-training assessments than those trained with the transparent eyewear. We adopted a broad methodological approach by using a variety of assessments and extensively piloting these measures. Findings from several of these assessments are reported here, and we discuss additional measures and future directions in the General Discussion.

With the exploratory nature of this project, we took an approach that would allow the data to speak to the possible mechanisms that might be affected by stroboscopic visual training. We hypothesized a priori that stroboscopic training would influence aspects of visual cognition related to temporal processing of the visual environment, and/or the allocation of attention, to appropriate visual elements. However, it is entirely possible that other aspects would be equally or more influenced. To begin to address this issue, in the present paper we report the results from three computer-based assessments that measured perceptual and attentional faculties hypothesized to benefit from stroboscopic training. These tasks include measures of central and peripheral motion sensitivity (motion coherence tests), distributed transient attention [useful field of view (UFOV) - dualtarget task], and distributed sustained attention (multiple-object tracking).

Before delving into the details of the current study, it is worth noting a few specific aspects of this research project. Primarily, by using battery-powered eyewear as our training tool we were able to conduct training outside of the laboratory and utilize athletic groups already engaged in highly interactive visual-motor activities. As well, because our research questions are the first steps of a much larger enterprise, we opted to take several experimental approaches that would afford us the best chance of measuring generalized training effects. First, we always administered the posttraining assessments immediately after the last training session in order to avoid potential loss of the trained effects over time. Second, we always compared our experimental Strobe participants to matched Control participants that engaged in the identical training regimen, but wore eyewear containing transparent lenses. Finally, in line with previous research indicating that sleep facilitates the consolidation of learned skills (e.g., Marshall and Born, 2007), we structured training so that it spanned multiple days and always included at least one night of sleep.

The primary goal of our training procedure was to have individuals perform tasks that actively engaged the visual-motor system (e.g., catching and throwing) under stroboscopic visual conditions. We were able to achieve this through two different types of training: in-lab and team-based (see Materials and Methods for full detail). In-lab training consisted of a ball catching game that was conducted in a controlled indoor environment. The in-lab training 
provided several benefits, as we were able to control the experimental timing and the training environment, and we were able to test Strobe and Control participants independently. However, in-lab training also had disadvantages in that it was logistically difficult to run multiple training sessions in the laboratory setting, and it presented limits on the nature of the physical activities that could be undertaken.

For the team-based training we partnered with campus athletic groups to administer our training regimen during their already established practice schedules. For the currently presented data we worked with the Duke University Varsity Football team and with the Duke Men's and Women's Club Ultimate Frisbee teams. This allowed us to provide training in highly engaging visualmotor activities as well as allowing up to 20 individuals to undergo training at the same time. The team-based training also had disadvantages, as the Strobe and Control participants were trained in the same practice sessions and therefore could be aware of the experimental condition assignments. By conducting both in-lab and team-based training regimens, we looked to overcome the shortcomings of each. Further, by using multiple training cohorts (in-lab, Club Ultimate Frisbee, and Football), we can compare performance to quantify any potential differences in training effects that are due to differences between these three different cohorts.

\section{MATERIALS AND METHODS PARTICIPANTS}

In-lab participants were recruited through campus advertisements and the Psychology department participant pool. Team-based participants were recruited through athletic trainers and team captains. Participants were advised not to participate if they had a history of seizures, migraines, or light sensitivity. The data reported here come from 157 participants. Each participant was compensated for the computer-based testing with either cash or with experiment participation credit in partial fulfillment of a Psychology department requirement. Voluntary informed consent was obtained for every session in accordance with the Duke University institutional review board.

\section{STUDY DESIGN}

Each participant was involved in two aspects of this study computer-based assessments and visual-motor training. The computer-based assessments were administered prior to training and immediately after the final training session. One group of participants also completed an assessment phase halfway through their training regimen (Ultimate Frisbee). Within each participant cohort (in-lab, Ultimate Frisbee, Football), participants were assigned to either the Strobe training condition or the Control training condition ${ }^{2}$. This was done randomly for the in-lab participants. For the team-based participants, experimental condition assignment was done pseudo-randomly to balance groups by athletic skill set (e.g., half of the football linemen were randomly assigned to the Strobe condition and half to the Control condition).

\footnotetext{
${ }^{2}$ For clarity we use the phrase "cohort" to refer to the different collections of training participants (i.e., in-lab, Ultimate Frisbee, Football) and the term "condition" to refer to the Strobe vs. Control training regimens.
}

Our experimental approach adhered to standard clinical treatment designs in which the null-hypothesis was that no difference existed between a treatment (Strobe) condition and a matched control condition following administration of the treatment. Typical null-hypothesis significance testing is applied to the data to determine if there are differences in performance on the computerbased assessments for the different experimental factors. Most notably, we use mixed-model ANOVAs with a within-subject factor of "Session" (pre-training vs. post-training), and betweensubject factors of "Condition" (Strobe vs. Control) and training "Cohort" (e.g., 2-day, Ultimate Frisbee, Football). To bolster and strengthen these analyses, we also computed Bayesian information criterion (BIC; Glover and Dixon, 2004; Wagenmakers, 2007) values. For each experiment we compared two models: model $\mathrm{H}_{0}$ (Constant) represents the null-hypothesis for the experiment, and model $\mathrm{H}_{1}$ [Constant $+($ Session $\times$ Condition $\left.)\right]$ represents the added explanatory power of the critical Session by Condition interaction term. The critical aspect of these analyses is that one can compare the change in BIC ( $\triangle \mathrm{BIC})$ between the two models to estimate which model is more likely, and how much more likely $(\lambda \mathrm{H}) . \lambda \mathrm{H}$ represents the approximate posterior probability of $\mathrm{H}_{1}$ over $\mathrm{H}_{0}$. If $\lambda \mathrm{H}$ favors model $\mathrm{H}_{0}$, this suggests that the null-hypothesis is correct and that the stroboscopic training did not affect performance. However, if $\lambda \mathrm{H}$ favors model $\mathrm{H}_{1}$, this would support a positive contribution of stroboscopic training. Moreover, it is possible to interpret these results in terms of statistical evidence for each hypothetical model (e.g., "weak," "positive," “strong” Raftery, 1995 Table 7).

\section{COMPUTER-BASED ASSESSMENTS}

Computer-based assessments were administered either via Dell Inspiron computers running Matlab R2010a and the Psychophysics Toolbox (http://psychtoolbox.org), or Apple Macintosh computers running OS9 and the PowerDiva software suite. All computers were attached to CRT monitors that were calibrated in order to assure that all visual stimuli were the same size regardless of slight differences in screen size. Monitors attached to the Dell computers were set to a $75-\mathrm{Hz}$ screen refresh and $1280 \times 1024$ resolution. Those attached to Macintosh computers were set to a 75$\mathrm{Hz}$ screen refresh and $800 \times 600$ resolution. Assessments for the inlab participants were collected in the Visual Cognition Laboratory at Duke University with one or two participants at a time. Teambased participants either completed the assessments in the same lab or in a dedicated computer lab in the football practice facility that could accommodate 1-10 participants at the same time. The order of the computer-based assessments was counterbalanced across participants, and the testing took 40-60 min to complete.

\section{STROBOSCOPIC TRAINING REGIMENS}

The activities engaged in during the stroboscopic training were tailored to each participant cohort, but importantly, the Strobe condition and the Control condition were always run in the same manner within each cohort. Prior to training, participants were instructed on how to operate the eyewear. They trained with the eyewear for a specified duration in each training session, as described for the three training cohorts below and summarized in Table 1. In general, training began at the fastest (i.e., easiest) 
Table 1 | Summary of training cohorts.

\begin{tabular}{lllll}
\hline Cohort & No. of sessions & Session length $(\mathbf{m i n})$ & Activities & No. of participants \\
\hline In-lab training & 2 or 4 & 27 & Catch (see Table 2) & 85 \\
Club ultimate frisbee & 4 & $20-28$ & Frisbee practice & 31 \\
Varsity football & 9 or 10 & $15-30$ & Speed and agility drills & 41 \\
\hline
\end{tabular}

strobe rate $(6 \mathrm{~Hz})$ and was made progressively harder by reducing the strobe rate (i.e., increasing the occlusion length) after five consecutive successful catches. This "leveling-up" was done until they reached a pre-specified level or time period. In longer training activities participants restarted at level 1 and repeated the process.

\section{In-lab: participants, training, and assessments}

The in-lab data presented in the current paper come from 85 participants. Of these participants, different individuals took part in the motion coherence thresholds task, the UFOV - dual-target task, and the multiple-object tracking task (for details see Overview of Training Participants for each). Eighty of the in-lab participants completed a 2-day protocol - they made two visits to the lab, and on the first day they completed the computer-based assessments then participated in a 27 -min training session (see Table 2). They returned to the lab within $48 \mathrm{~h}$ to complete a second training session and then were re-administered the computerbased assessments. A second set of five participants underwent a longer multi-day training protocol. These participants completed a computer-based assessment and 27-min training on the first day, and then returned to the lab and participated in eight more training sessions over 19 days. Following the fourth and eighth sessions they were re-administered the computer-based assessment immediately following their training sessions. We focus here only on the assessments collected after the fourth training session to make the data maximally comparable to the other participants' (e.g., Ultimate Frisbee). In-lab training was conducted by members of the research team in a location adjacent to the computer assessment room. The space consisted of a well-lit 20-foot hallway. Consistent lighting was maintained by covering a large window at one end of the hallway.

\section{Men's and women's ultimate frisbee: participants, training, and assessments}

Members of the Men's and Women's Club Ultimate Frisbee teams participated in a multi-day testing and training study. Computer assessments were administered in the Visual Cognition Laboratory before training, after the fourth training session, and then again after the eighth training session. Thirty-one club members participated, with 16 assigned to the Strobe training condition and 15 to the Control training. Training sessions $1-3$ and 5-7 were conducted at the teams' normal Tuesday and Thursday evening practices and consisted of typical Ultimate Frisbee activities involving passing and throwing drills in both stationary and running situations. The stroboscopic frequency level for the Strobe participants was initially set to level 1, and the participants decreased the strobe rate after five consecutive catches until they reached a maximum at level 6. The rate was reset to level 1 after each of four 6-min
Table 2 | Twenty-seven minute in-lab training protocol.

\begin{tabular}{ll}
$\begin{array}{l}\text { Duration } \\
\text { (min) }\end{array}$ & Activity \\
\hline 1 & $\begin{array}{l}\text { Warm-up: Eyewear on head or around neck; forward facing } \\
\text { catches }\end{array}$ \\
10 & $\begin{array}{l}\text { Forward facing catches: Start at level 1, decrease rate (i.e., } \\
\text { "level up") every } 5 \text { consecutive catches until level } 6 .\end{array}$ \\
5 & $\begin{array}{l}\text { Forward facing catches, with variable speeds: Start at level 2, } \\
\text { level up every } 5 \text { consecutive catches until level } 6 .\end{array}$ \\
5 & $\begin{array}{l}\text { Turn and catch: Experimenter calls out "ball" as they released } \\
\text { ball. Participant turns to face experimenter and catch ball. }\end{array}$ \\
& $\begin{array}{l}\text { Start at level } 2 \text { and level up every } 5 \text { consecutive catches until } \\
\text { level } 6 .\end{array}$ \\
& Forward facing catches: At highest level reached during the \\
& first 10-min section. \\
& Cool-down: Eyewear on head or around neck; forward facing \\
& catches
\end{tabular}

phases, for a total of $24 \mathrm{~min}$ in each training session. The Control participants did everything exactly the same as the Strobe participants, including pressing the buttons for leveling-up, but their lenses remained transparent throughout. Training sessions 4 and 8 were conducted outside of the Visual Cognition lab (in participant-pairs or with a lab member). These sessions lasted $24 \mathrm{~min}$ and were modeled after the teams' sessions. These two sessions were completed immediately before the mid-training and post-training computer-based assessments. To best compare these data with those from our other participant groups, we focus on the pre-training and mid-training assessments. Each participant completed two computer-based assessments: the multiple-object tracking task and the motion coherence threshold task. Task order was counterbalanced across participants.

\section{Varsity football: participants, training, and assessments}

Members of the Football team participated in a multi-day testing and training study. Computer-based assessments were administered before and after training. Physical measures were also collected, but are not reported here. All testing and training were conducted in the football-training building on campus. Forty-one student-athletes participated ( 21 in the Strobe condition and 20 in the Control condition), and one participant assigned to the Strobe condition did not complete the final post-test (his data are not considered in the analyses).

Participants completed 9 or 10 total training sessions. All but the final session were conducted during normal practice sessions, which were led by the team strength and conditioning coaches. These sessions were completed within a 12 -day period. Training 
consisted of warm-up and agility drills, with variability in timing (between 10 and $30 \mathrm{~min}$ ) and activities across sessions. The stroboscopic frequency level for the Strobe condition varied across training sessions - the early training sessions were done at a single rate and the later sessions involved leveling-up from faster to slower rates at set time intervals between organized drills. Overall, Strobe condition participants primarily experienced levels 2-4 (5-3 Hz). The Control participants experienced everything exactly the same as the Strobe participants, including pressing the buttons on the eyewear for leveling-up, but their eyewear remained transparent throughout.

The final training session took place within 7 days of the previous training, and was conducted in conjunction with the posttraining computer-based assessments. This training session lasted $30 \mathrm{~min}$ and was divided into three components of visual-motor control activities. These included $14 \mathrm{~min}$ of catching a tennis ball thrown off a wall by a partner, 10 min of football throwing and catching, and 6 min catching a heavy ball dropped by a partner. The Strobe participants followed a systematic leveling-up protocol in which the stroboscopic rate got slower and harder over time within each activity. The Control participants did the same activities but with the transparent lenses.

The computer-based assessments were administered before and after the training regimen. The pre-training phase took approximately $75 \mathrm{~min}$ and consisted of a physical measure (not reported) and two computer-based assessments; the UFOV - dual-target task and the multiple-object tracking task. The order of the two assessments was counterbalanced across participants. The posttraining phase was identical, but included a 30-min training session immediately prior to the computer-based assessments.

\section{RESULTS}

\section{OVERVIEW}

Three experiments are presented here. In Experiment 1, we assessed possible changes in motion sensitivity using a motion coherence threshold task. Experiment 2 assessed peripheral vision and dual-task attentional abilities using a UFOV paradigm. Experiment 3 assessed sustained attention via a multiple-object tracking task. We present each experiment and then discuss the broader implications of our findings in the General Discussion.

\section{EXPERIMENT 1: MOTION COHERENCE THRESHOLDS \\ Experimental motivation}

We hypothesized that stroboscopic training could influence aspects of visual cognition related to the temporal processing of the visual environment. One fundamental aspect of temporal processing is the ability to detect subtle motion cues. We therefore tested for training-related changes in sensitivity to subtle motion cues using a well-established motion coherence threshold task that estimates the threshold at which individuals can detect coherent motion in a two alternate forced-choice design (Snowden and Kavanagh, 2006).

\section{Stimuli and procedure}

The stimuli, depicted schematically in Figure 1A, consisted of square $10^{\circ} \times 10^{\circ}$ dot fields with a $5 \%$ dot density and individual dot elements spanning $4 \mathrm{~min}$ of visual angle. On each screen refresh ( $13.33 \mathrm{~ms})$, dots moved $10 \mathrm{~min}$ of visual angle, and $1 \%$ of the individual dots were replaced by new, randomly positioned dots (this led to $60 \%$ of the dots within a trial being generated anew and $40 \%$ remaining for the duration of the trial). Dot contrast was set at $90 \%$
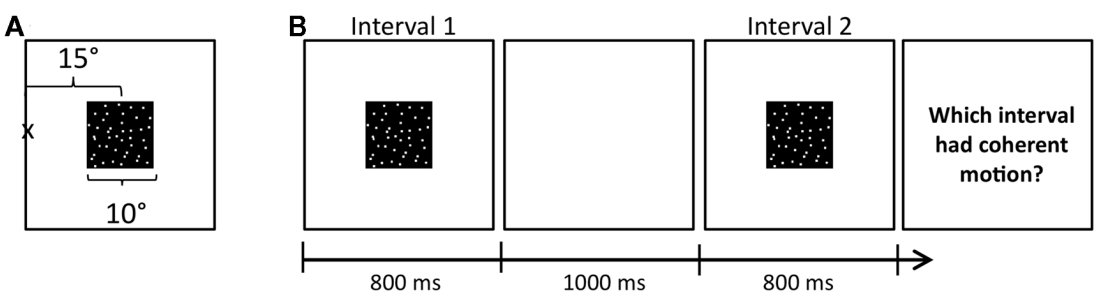

C

Central Task Coherence Thresholds

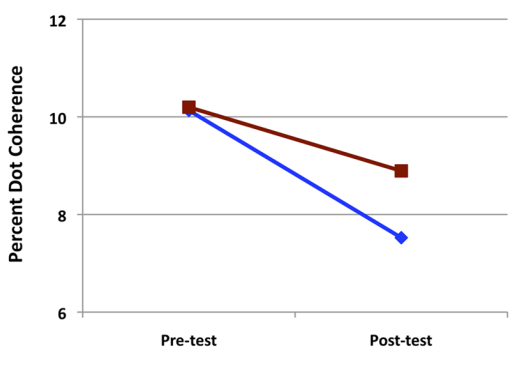

Strobe Eyewear

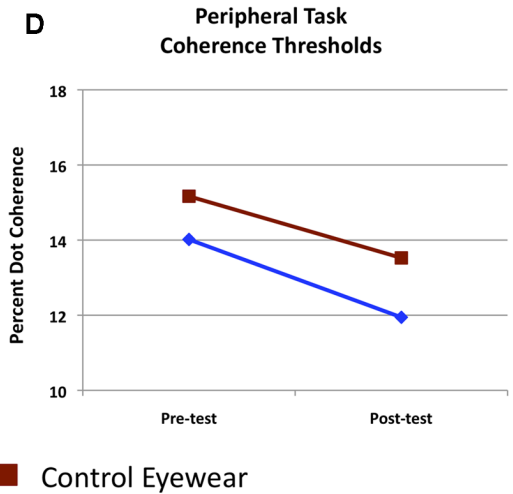

FIGURE 1 | Motion coherence task and results. Schematic illustration depicting (A) the relative size and position of the dot stimulus, and (B) the two-interval forced-choice procedure. Pre- to post-test motion coherence thresholds improved more for the Strobe (blue) than the Control (red) participants for the central task (C) but not for the peripheral task (D). These data are collapsed over the non-significant factor "Cohort." 
of the monitor maximum and appeared as white dots on a black background. The field of moving dots was presented in two temporally separate intervals (Figure 1B). One interval contained 100\% random dot motion directions, and the other interval had some percentage of the dots moving coherently in a single horizontal direction, with the remaining dots moving in random directions. The motion stimuli were separated by a 1-s inter-stimulus-interval, and successive trials automatically initiated following the participants previous-trial response. In separate experimental blocks these stimuli were presented centrally or peripherally, with fixation $15^{\circ}$ to the left of the center of the dot field. The order of the central and peripheral blocks was counterbalanced across participants, and the stimuli were viewed from approximately $57 \mathrm{~cm}$ without head restraint.

On each trial, participants viewed two $800 \mathrm{~ms}$ temporally separate intervals of moving dot fields and were to report which of the two intervals contained coherent motion (there was always one interval with coherent motion and one without, the order was randomly determined on each trial). The motion coherence started at $24 \%$ (i.e., for the first trial, the motion field that contained coherent motion had $24 \%$ of the dots moving in a single direction, either left or right, with the other $76 \%$ moving randomly). Following a correct response, the coherence was reduced by $2 \%$ (making the next trial harder) and following an incorrect response it was increased by $4 \%$ (making the next trial easier). This 2-down/1-up staircase procedure estimates the percentage of dot that must move coherently for each participant to produce $82 \%$ correct performance. Individual participant threshold values were computed as the average of three staircases (excluding an initial practice trial).

\section{Overview of training participants}

Sixty-seven participants from the Ultimate Frisbee (31), 4-day in-lab (5), and 2-day in-lab (31) training groups (see Materials and Methods for training details) performed this task. Four individuals from the Ultimate Frisbee cohort were excluded for not completing the second testing session, leaving 63 individuals in the final analysis. All 63 of these participants completed the central viewing task version, while 40 of the 63 were also run on the peripheral task version. The Ultimate Frisbee and 4-day in-lab participants underwent identical testing/training schedules, and their coherence thresholds did not differ for either the pre $(t=0.874$, $p=0.394)$ or post $(t=0.268, p=0.792)$ tests, therefore these two participant pools were combined into the same Cohort factor.

\section{Motion coherence task results}

While all participants completed the central viewing version of the task, only a subset completed both the central and peripheral versions. As such, we present the data separately.

Central viewing coherence thresholds. A mixed-model ANOVA performed on the Session $\times$ Cohort $\times$ Condition coherence threshold data for the central viewing task revealed a significant within-subject main effect of Session $[\mathrm{F}(1,59)=41.97, p<0.001]$ and a significant Session $\times$ Condition interaction $[\mathrm{F}(1,59)=4.13$, $p=0.047]$. While there was a significant between-subject main effect of Cohort $[\mathrm{F}(1,59)=7.74, p=0.007]$, indicating that the 2-day in-lab participants had overall higher thresholds, this factor did not interact with Session $[\mathrm{F}(1,59)=1.319, p=0.255]$, and there was no three-way interaction $[\mathrm{F}(1,59)=2.475, p=0.121]$, indicating that the training cohorts did not differ with regard to any other experimental factors. As depicted in Figure 1C, pre-test thresholds for the Strobe participants $(10.13 \%)$ and the Control participants $(10.20 \%)$ did not statistically differ $(t=0.067$, $p=0.946)$, but after training the Strobe participants $(7.52 \%)$ achieved lower threshold performance than the Control participants $(8.89 \%)$. This re-test improvement translates to a $25.7 \%$ improvement in thresholds for Strobe participants from pretraining to post-training and a $13.3 \%$ improvement for Control participants.

The above statistical testing provides evidence that stroboscopic training improved the detection of coherent motion, but it does not provide a metric of the relative magnitude of the improvement. To address this we employed BIC analyses. Examining the change in the BIC between two models gives a measure of which best fits the data. In this framework, we compared two models: the $\mathrm{H}_{0}$ model (Constant) which represents the null-hypothesis that there are no differential effects of stroboscopic training, and the $\mathrm{H}_{1}$ model [Constant $+($ Session $\times$ Condition $)$ ] which includes the Session by Condition interaction. If the stroboscopic training produced a robust pre- to post-training benefit we would expect the data to better fit the more complex $\mathrm{H}_{1}$ model, which includes the Strobe and Control treatment conditions, than the null-hypothesis $\mathrm{H}_{0}$ model. Contrasting the models indicated that the data provided support for the $\mathrm{H}_{1}$ model over the $\mathrm{H}_{0}$ model $\left(\Delta \mathrm{BIC}=0.120, \lambda \mathrm{H}_{1}=0.5150\right)$. This model test indicates that the data provide "weak" evidence (Raftery, 1995) that stroboscopic training improved visual motion sensitivity. In conjunction with the ANOVA data above, this analysis suggest stroboscopic training in the current experiment can weakly improve motion sensitivity for centrally presented stimuli.

Peripheral viewing coherence thresholds. A mixed-model ANOVA performed on the peripheral motion coherence thresholds demonstrated only a significant main effect of Session $[\mathrm{F}(1,36)=8.963, p=0.005]$. There were no significant interactions with Cohort and Condition. Control participants had slightly elevated coherence threshold relative to Strobe participants (see Figure 1D), but these between-subject differences were not significant $[\mathrm{F}(1,36)=1.427, p=0.240]$. BIC analyses contrasting the $\mathrm{H}_{0}$ model (Constant) and the $\mathrm{H}_{1}$ model [Constant $+($ Session $\times$ Condition $)]$ indicated greater support for the $\mathrm{H}_{0}$ model over the $\mathrm{H}_{1}$ model $\left(\Delta \mathrm{BIC}=0.982, \lambda \mathrm{H}_{1}=0.3796\right)$. This suggests that the data are most consistent with the null-hypothesis that stroboscopic training did not affect motion sensitivity for peripherally presented stimuli.

\section{Summary}

The results of Experiment 1 demonstrate that one form of motion sensitivity, dot motion coherence thresholds, shows a significant re-test improvement for the stroboscopic training over the matched control training. However, at least for the amount of stroboscopic training employed here, this improvement was modest at best, with only a weak change in the effect size. In addition, 
this benefit only occurred for centrally viewed stimuli, and not for the peripherally viewed stimuli.

\section{EXPERIMENT 2: USEFUL FIELD OF VIEW - DUAL-TARGET TASK Experimental motivation}

We hypothesized that stroboscopic training could influence aspects of visual cognition related to transient attention. The UFOV task is an established measure that characterizes the extent of the visual field from which information can be extracted in a single glance (Ball and Owsley, 1991). UFOV effects predict performance in complex tasks like driving (Myers et al., 2000), and practice on UFOV tasks has been shown to reduce the detrimental effects of divided attention (Roenker et al., 2003; Richards et al., 2006). In the present study, we implemented a specific dual-target version of the UFOV task wherein participants had to detect a target in the periphery while also making a decision about a centrally presented letter. This addition of a central letter target was done for two reasons. First, pragmatically, this forced the participants to maintain central fixation since both stimuli were presented simultaneously and for only a very brief duration. Second, UFOV performance has previously been shown to be influenced by attentional load when participants have to divide their attention in a dual-task setting (e.g., Sekuler et al., 2000) and therefore implementing this version of the task allows us assess the effects of stroboscopic training under demanding dual-task conditions.

\section{Stimuli and procedure}

Every trial consisted of four successive displays viewed from approximately $31 \mathrm{~cm}$ without head restraint. This relatively close distance to the screen was required to achieve a sufficiently broad $\left(30^{\circ}\right)$ viewing angle for the experimental stimuli. Head position was maintained by periodically measuring the distance, and by encouraging the participants to stay in place. The first display, which lasted for $1500 \mathrm{~ms}$, had eight equally spaced arms radiating out from a central $3.0^{\circ}$ circle (see Figure $2 \mathrm{~A}$ ). The second display lasted for $\sim 90 \mathrm{~ms}$ (see below) and contained the dual-target array. Here, the eight radial arms remained with the addition of three unfilled $1.0^{\circ}$ circles placed on each of the radial arms at $10^{\circ}$, $20^{\circ}$, or $30^{\circ}$. On each trial, 1 of these 24 locations was selected as the target location and filled in black. Additionally an uppercase or lowercase letter appeared within the center fixation circle. All letters used were selected to have clear differences between upper case and lowercase (e.g., Q,q; R,r). Targets were counterbalanced such that for each subject every location was presented once with an upper case and once with a lower case letter in each block. The third display, which lasted for $200 \mathrm{~ms}$, contained 1 of 12 randomly selected white-noise mask screens. The fourth display, which remained until response, was the response screen consisting of the same eight radial arms with the outline of a circle at each location where a target might have appeared. Participants were to make a mouse click on the location where they believed the target was located during the test display. Participants then indicated via a keyboard button press if the letter displayed during the test phase was an uppercase or lowercase letter. The trial ended once a response had been made and the next trial was initiated with a key press. Participants completed 6 blocks of 48 trials each. The first block was considered practice and not analyzed.

The length of the stimulus presentation for in-lab participants was held constant at $93.3 \mathrm{~ms}$. However, to adjust for possible differences in baseline abilities in varsity athletes, the length of

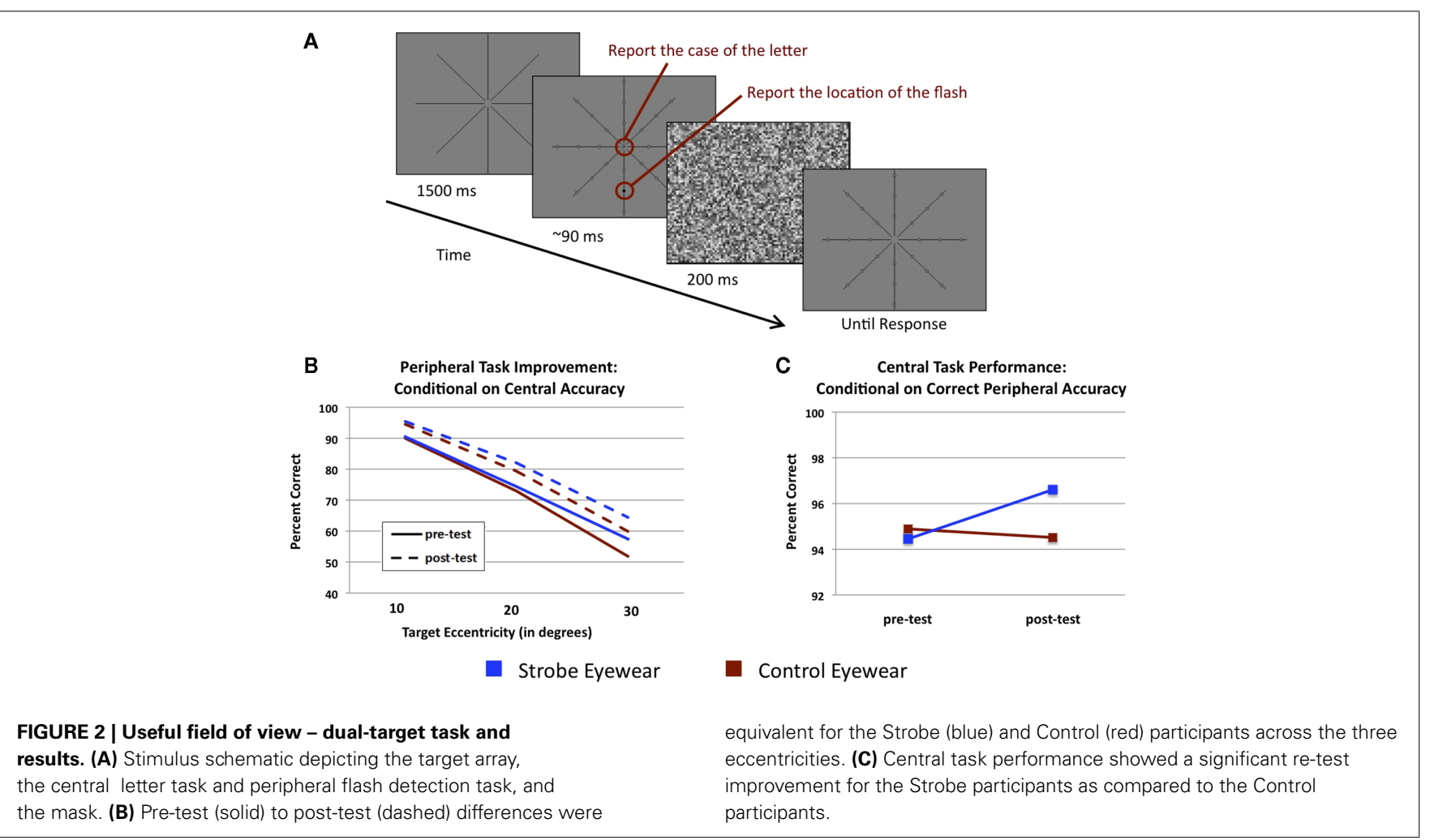


the stimulus presentation for the Football team was set for each individual with a titration procedure prior to the first test. In this 2-3 min block, targets only appeared at the $20^{\circ}$ eccentricity. The length of stimulus presentation began at $106.7 \mathrm{~ms}$ and decreased by $13.3 \mathrm{~ms}$ (i.e., became more difficult) for each correct response and increased by $13.3 \mathrm{~ms}$ (i.e., became easier) for each incorrect response. After the staircase reversed direction on three consecutive trials, the shorter of the last two measurements was used as the stimulus duration for both the pre- and post-tests. If the final duration was longer than $125 \mathrm{~ms}$, then $125 \mathrm{~ms}$ was used as the stimulus duration. The average duration was $88 \mathrm{~ms}(\mathrm{SD}=30 \mathrm{~ms})$.

\section{Overview of training participants}

Participants who performed this task came from the Football team $(N=41)$ and 2-day in-lab $(N=34)$ training groups (for details refer to Materials and Methods). Of the 75 total participants recruited, two were excluded for not completing the post-test (1 Football; 1 in-lab) and four were excluded for failing to follow instructions during the post-test (all from Football), leaving 69 individuals in the final analysis.

\section{Task results}

To assess re-test improvements due to stroboscopic training, we first analyzed percent correct performance on the central and peripheral tasks under conditional dual-task performance. That is, given accurate performance on the central task, what was the peripheral task accuracy, and vice versa. We performed mixed-model ANOVAs with the within-subject factors of Session (pre-training, post-training) and Eccentricity $\left(10^{\circ}, 20^{\circ}, 30^{\circ}\right)$, and the between-subject factors of Cohort (Football, 2-day inlab) and Condition (Strobe, Control). These analyses revealed no significant Session $\times$ Condition interaction at any eccentricity ( $p$ 's $>0.1$ ) for peripheral performance, conditional on correct central task performance (Figure 2B). While there was a main effect of eccentricity $[\mathrm{F}(2,130)=146.99, p<0.001]$, but this factor did not interact any other factor $(p>0.1)$. However, analyses of central (letter) task performance, conditional on correct peripheral task performance (Figure 2C), revealed a significant Session $\times$ Condition interaction $[\mathrm{F}(1,130)=7.81, p=0.007]$ and a Session $\times$ Eccentricity interaction $[\mathrm{F}(2,130)=3.22, p=0.043]$. No other main effects or interactions reached significance. Post hoc comparisons showed a significant re-test effect for the Strobe participants $[t(34)=2.65, p=0.012]$, but no significant difference between pre- and post-test for the Control participants $[t(33)=0.93, p=0.361]$, indicating that the Session $\times$ Condition interaction was driven by re-test improvements in the Strobe participants. The absence of any interaction with Cohort reveals that similar patterns of effects were seen for both the Football and in-lab participants. In fact, when tested separately (which reduces the overall power by using roughly half of the data) the Football-training group maintained a significant Session $\times$ Condition interaction $[\mathrm{F}(1,34)=4.33, p=0.045]$, and the in-lab participant group trended in the direction of the overall effect $[\mathrm{F}(1,31)=3.67, p=0.065]$.

As in Experiment 1, we complemented the analysis of variance with the use of BIC analyses (see Experiment 1 results for details). For the central task data conditional on correct peripheral performance, we compared two models: $\mathrm{H}_{0}$ (Constant) and $\mathrm{H}_{1}$ [Constant $+($ Session $\times$ Condition $)]$. This analysis provided support in favor of the $\mathrm{H}_{1}$ model $\left(\Delta \mathrm{BIC}=-2.426, \lambda \mathrm{H}_{1}=0.7708\right)$, indicating that the data provide positive evidence that stroboscopic training does improve visual motion sensitivity.

To determine if the pattern of effects seen for the conditional analyses was driven by the fact that this was a dual-task paradigm, we re-ran the analyses on the percent correct data, no longer conditioned on correct performance on the other task. That is, we looked at the peripheral task performance regardless of central task accuracy, and vice versa. The pattern of results was unchanged. There was no training effect in the peripheral task for any eccentricity (all $p$ 's $>0.1$ ), and there was still a significant Session $\times$ Condition interaction for central task performance $[F(1,65)=5.55$, $p=0.022]$. As well, the BIC analyses remained the same, with support for the $\mathrm{H}_{1}$ model $\left(\triangle \mathrm{BIC}=-1.366, \lambda \mathrm{H}_{1}=0.6644\right)$, suggesting that the pre- to post-training differences were likely driven by differences in the Strobe and Control conditions.

\section{Summary}

In this task we observed significant dual-task performance improvements for the Strobe training participants, but not the Control participants, for the central attentional task. The Strobe participants significantly improved their accuracy performance by $2.21 \%$ from pre- to post-training at the central task, while the Control participants non-significantly worsened by $0.83 \%$. The Strobe participants' small but significant improvement suggests that stroboscopic training can increase the ability to quickly process visual information in the central visual field. No differences were found for either group in the peripheral attention task. In addition, the lack of Cohort effects suggests that 2 days of training may be sufficient to produce stroboscopic training improvements in central attention, and that differences in the specific details of the training regimen may not be critical.

\section{EXPERIMENT 3: MULTIPLE-OBJECT TRACKING Motivation for the task}

We hypothesized that Strobe training could influence aspects of visual cognition related to sustained attention, especially the ability to divide attention over space. To test this, we performed a version of the well-established multiple-object tracking task (Pylyshyn and Storm, 1988).

\section{Stimuli and procedure}

This task examined participants' ability to divide their attention in order to track multiple-objects amongst identical distracter items (Figure 3A). Each trial began with $15,1^{\circ}$-diameter dots presented in a $17.5^{\circ} \times 17.5^{\circ}$ box centered on the screen and were viewed from approximately $57 \mathrm{~cm}$ without head restraint. In the middle of the box was a $1^{\circ} \times 1^{\circ}$ fixation cross. Participants were instructed to keep their eyes on the fixation cross throughout the entire trial and use their full range of central and peripheral vision to perform the task. The trial began when a subset of the dots would blink for $1 \mathrm{~s}$, and these were the target dots that were to be tracked. After the target dots stopped blinking, all dots moved around the screen pseudo-randomly and independently for $8 \mathrm{~s}$, avoiding collisions with the boundary and other dots. Once the motion stopped, one 


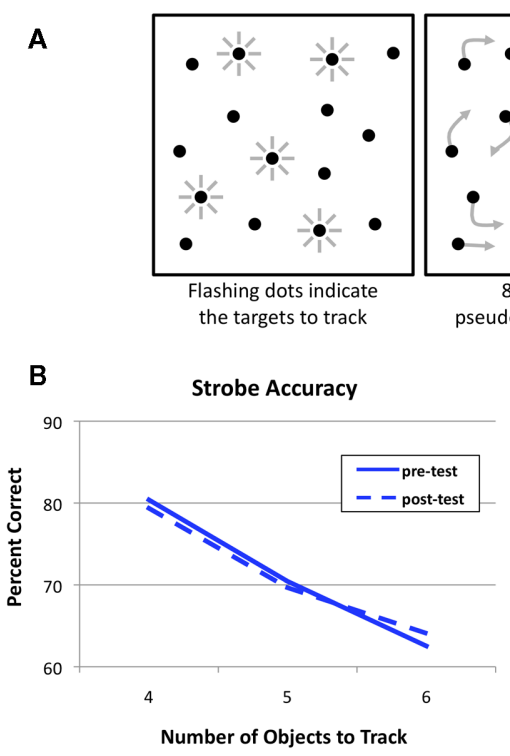

FIGURE 3 | Multiple-object tracking task and results. (A) Stimulus schematic showing the five blinking dots to indicate the set of objects to be tracked, the initiation of their movement, and the probe item illuminated in yellow. Participants' task was to report if the yellow probe was among the set of objects to be tracked. (B) Strobe and (C) Control accuracy is shown as a function of the number of items tracked for the pre-test (solid) and post-test (dashed). No differences between pre- and post-test accuracy were observed for either training condition. dot would become yellow and participants were to report with a key press whether or not this dot was a target. On all trials there was a $50 \%$ chance of the yellow dot being a target. Every participant was given a practice block of four trials. The number of trails and targets tracked varied across participant groups; the in-lab participants tracked between 4 and 6 objects on a given trial and performed 24 trials in each of 7 blocks, the Football group tracked between 4 and 7 objects on a given trial and performed 22 trials in each of 7 blocks, and the Ultimate Frisbee group tracked between 3 and 6 objects on a given trial and performed 24 trials in each of 7 blocks. The number of targets tracked was always counterbalanced across trials. Because not all participants received the "track 3" and "track 7" conditions, these two levels are not considered further. All analyses are therefore done with the factor "Objects" containing only trials with 4-6 tracked objects.

\section{Overview of training participants}

Participants who performed this task came from the Football $(N=41)$, Ultimate Frisbee $(N=31)$, and 2-day in-lab $(N=38)$ training cohorts (for details refer to Materials and Methods). Six participants (5 Ultimate Frisbee and 1 Football) were excluded for failure to complete the full training/testing protocol and 16 other participants (12 Football, 4 in-lab) were excluded for poor performance (being less than $60 \%$ accurate on the condition with the fewest number of items to be tracked), leaving 88 individuals in the final analysis.

\section{Task results}

We performed a mixed-model ANOVA with the within-subject factors of Session and Objects, and the between-subject factors of Condition and Cohort on the percent correct data. This analysis revealed that while there was an overall effect of number of objects to track $[\mathrm{F}(2,164)=150.153, p<0.001]$, there was no effect of Session (i.e., no re-test effect): $\mathrm{F}(1,164)=0.007, p=0.933$. The between-subject main effect of Condition was not significant $[F(1,82)=0.006, p=0.692]$, nor was the interaction between Session and Condition $[\mathrm{F}(1,164)=0.029, p=0.864]$, indicating that the Strobe and Control participants did not differ in performance Figures 3B,C. There was however a main effect of Cohort $[F(2,82)=3.302, p=0.042]$ with the Ultimate Frisbee team showing the best, 2-day in-lab intermediate, and Football showing the worst performance. Cohort did not interact with any other factors, however, suggesting that the three groups responded similarly to the other experimental manipulations.

Bayesian information criterion analyses (see Experiment 1 results) were conducted with two models: $\mathrm{H}_{0}$ (Constant) and $\mathrm{H}_{1}$ [Constant $+($ Session $\times$ Condition $)] . \mathrm{H}_{0}$ was strongly favored over $\mathrm{H}_{1}\left(\Delta \mathrm{BIC}=4.446, \lambda \mathrm{H}_{1}=0.0977\right)$. This indicates that the data are most parsimonious with the null-hypothesis model and that there was not a Session by Condition interaction related to behavioral performance.

\section{Summary}

The goal of this task was to examine whether or not stroboscopic training could improve sustained attention to multiple moving objects. This skill has clear connections to sports (e.g., tracking teammates and opponents on the basketball court or football field), and we had predicted that the stroboscopic experiences would lead to enhancements in sustained attention. Likewise, previous work has demonstrated that individuals trained with first-person shooter video games revealed improved accuracy at this task (Green and Bavelier, 2006b). As such, we were somewhat surprised to not find a significant effect here. Consistent with Green and Bavelier we did not find re-test improvements for 
our Control participants, however no re-test improvement was observed for the Strobe participants. Whereas action video game playing may improve the ability to track multiple-objects, stroboscopic training does not seem to impact this sustained attention task.

\section{DISCUSSION}

If people are forced to operate in an impoverished visual environment, might their visual abilities improve once they return to a normal environment? The goal of the current research program was to ask this question by determining if visual-motor training under stroboscopic visual conditions produced any generalizable learning to other untrained domains. To begin this exploration, we measured a range of computer-based visual cognitive abilities to determine which, if any, would reveal generalized changes in performance.

In the current study we found two visual cognitive measures that showed generalized stroboscopic training effects and several that did not. We observed lowered thresholds for detecting coherent motion in the center of the visual field, but not in the periphery. This effect was relatively weak, suggesting that the limited training employed here might only produce mild generalized benefits for motion sensitivity. Nevertheless, it is still noteworthy that playing catch in a stroboscopic environment can produce measureable differences on an orthogonal computer-based assessment of motion perception. Participants who underwent stroboscopic training were also more accurately able to report briefly presented stimuli in a dual-task setting that required broadly distributed spatial attention, but again, only for central presentations. This effect was somewhat stronger, suggesting a more robust transfer from training to test. These initial findings indicate that aspects of transient visual perception or attention may be enhanced due to training. No improvement, however, was observed for multiple-object tracking, indicating that sustained attention may not be impacted. The lack of uniform re-test improvements across tasks, or across the visual field, indicates that benefits do not transfer to all aspects of visual cognition, and suggests that there might be a specific profile to the generalization of stroboscopic training effects.

\section{TASKS AND MECHANISMS}

The current work serves as an initial foray into exploring whether generalized visual and attentional benefits can be produced through stroboscopic training. Our long-term objective is to establish what, exactly, is altered through stroboscopic training so that we can better understand the malleability of visual cognition more broadly. Here, we found both positive and null results, which set the stage for future research which can narrow in on the underlying mechanisms that might be affected. The three experiments reported here have begun this process, yet much remains to be uncovered.

While it is premature to delineate exactly what mechanisms underlie the observed generalized training benefits (and the observed null effects) some preliminary conclusions can be drawn. First, the observed improvement in motion sensitivity suggests that stroboscopic exposure alters spatiotemporal integration. Since most models of visual motion processing (e.g., Van Santen and Sperling, 1985) assume that local spatiotemporal correlations and subsequent integration occurs at a fairly low level in the visual hierarchy, it is possible that these effects may reflect some type of early sensory gain change. Second, while the UFOV task from Experiment 2 is typically employed to measure the extent of visual space from which information can be extracted in a brief glance, the current instantiation is most informative for its dualtask aspects. We observed that stroboscopic training only manifest in changes to central task performance, yet peripheral accuracy was quite high for these very briefly presented stimuli indicating attention was successfully distributed to the entire scene. Therefore the present findings suggest that stroboscopic training might improve processing of transient stimuli when attention is broadly allocated. Third, the lack of any training effects for stimuli presented in the periphery for either Experiments 1 or 2 suggests that the stroboscopic training employed here might not affect the spatial distribution of attention. These null results do not prove this definitively, but no hint of a widening of spatial attention was observed. Finally, the lack of an effect for the multiple-object tracking task (Experiment 3), suggests that the stroboscopic training might not affect sustained spatial attention abilities.

It is beyond the scope of the current paper, but we have other experimental tasks that, when combined with the current findings, might offer a means to further delineate the mechanisms influenced by stroboscopic training. For example, we have preliminary data from a visual sensory memory task and a flash-lag experiment that might provide insight into whether rapid sensory memory or temporal estimation processes are influenced. Additional important questions, such as how long does the learning last, how much training is needed to produce learning, and what particular strobe rates are most effective, will also provide valuable insight into the particular mechanisms that are affected. It is also worth noting, that a limited amount of data was collected on physical measures with the football-training cohort to see if any direct visual-motor learning occurred (i.e., testing on the measures that are trained). Our limited data were not sufficient to speak to this important question, but other recent research is addressing whether specific learning effects can result from stroboscopic training (Smith, 2011).

\section{RELATIONSHIP TO EXISTING LEARNING LITERATURE}

While there are many examples of specific perceptual learning effects (Fine and Jacobs, 2002), there are relatively few findings wherein practice at one task reveals improvements on another task. Recent years, however, have seen an expansion in the number and the scope of generalized learning effects described, and the current results complement and extend this literature. One field that is currently exploring generalized learning effects is the study of the cognitive effects of video game playing. Based on a rapidly growing set of empirical and computational studies, it is suggested that extensive experience with action video games can improve an individual's ability to form templates for, or extract the relevant statistics of, the task at hand (Green et al., 2009, 2010). Here we demonstrate that stroboscopic training might be another means of producing generalized improvements. However, video game playing and stroboscopic training do not produce identical results. For example, video game playing has been found to improve multipleobject tracking abilities (Green and Bavelier, 2006b), but here no 
such benefits were observed. Thus, more work is needed to compare and contrast the specific benefits of each training procedure, and such work should also help reveal the underlying mechanisms of each. Questions remain about the nature of video game training (Boot et al., 2011), so it is important to expand the study of potential tools for producing generalized learning effects.

Expertise can be thought of as an end-goal of training, and a natural question is whether gained expertise leads to generalized improvements across a variety of domains or to specialized learning specific to the expert's realm. Some lines of evidence suggest that experts are better than novices at processing information within their area of expertise, and that these may be generalized benefits. For example, there is evidence from eye tracking studies that expert athletes have more precise eye movements than nonathletes and are better able to focus on the most relevant cues (i.e., they fixate fewer targets, make fewer eye movements, and have longer fixations than non-athletes; (Mann et al., 2007). Recent studies have also provided some initial evidence that improved smooth pursuit eye movements are enhanced for both video game players and individuals who frequently engage in sporting activities (e.g., playing and watching; Koopman et al., 2011; Tsoi et al., 2011), further suggesting that generalized transfer is possible in both domains.

However, there are also lines of research that suggest that expertise only produces specific learning benefits. For example, when shown still shots of a soccer game, expert soccer players are better able to remember the positions of the players on the field (Mann et al., 2007), yet this benefit appears to not be a form of generalized learning since experts perform no better than novices if the players in the still shots are randomly organized. Similarly, grand master chess players can recall precisely the board position of pieces during a structured game, but fare no better than novices with randomly organized pieces (Simon and Chase, 1973). It is thought that such snap-judgment recognition results from better perceptual faculties, allowing expert to focus more on strategy, and resulting in better performance. The benefits found in the current studies are likely not strategic (see discussion of the role of motivation below) and may reveal true generalized learning.

\section{EXPERIMENTAL APPROACH AND MOTIVATIONAL EFFECTS}

In the current study we contrasted performance between experimental participants and a set of matched control participants, allowing us to disassociate test-re-test effects (i.e., how much improvement resulted simply from taking the test a second time) from generalized training that resulted from stroboscopic exposure. Nonetheless, other factors not related to stroboscopic training per se, could have influenced the outcome of our betweencondition comparisons. In particular, participant motivation has been widely shown to influence performance (Adcock et al., 2006). Given that our team-based training participants were able to see both the Strobe and Control versions of the eyewear, there is the potential for concern that the Strobe participants may have been more motivated, which could cause them to take a more active interest in their performance than the Control participants. This effect, dubbed the Hawthorne effect (Lied and Karzandjian, 1998), is well-known concern for these sorts of experimental situations.
Although this initial exploratory study of generalized stroboscopic training cannot rule out motivation entirely, three factors dampen these concerns. First, efforts were taken, both for the inlab and team-based training groups, to conceal the experimental objectives. Both versions of the eyewear, stroboscopic and transparent, were presented as experimental manipulations that may or may not affect performance, and terms such as "experimental" and "control" were never used with the participants. As well, both groups used the buttons on the side of the eyewear to "level up" based on regular time intervals or on successful performance. Second, the in-lab training participants were trained and tested individually and therefore had no knowledge of their condition assignment, or the presence of the other condition. Notably, the in-lab and the team-based participants appear to show similar effects - these groups produced consistent patterns of results for both the UFOV-DT and the multiple-object tracking tasks. Finally, had motivation been contributing to the condition differences, we would expect global benefits for the Strobe condition participants. However, the effects were not uniform across tasks or even within tasks. While we expected to find benefits of stroboscopic training for the multiple-object tracking task, none were found. Previous work from the video game playing literature using a similar paradigm found no test-re-test benefits for a control group, but clear benefits for the experimental group (Green and Bavelier, 2006b). Here we found no training effects for either. As well, while we found improvements in motion coherence thresholds for central stimuli in Experiment 1 and the letter discrimination for central stimuli in Experiment 2, no benefits were found for peripheral stimuli. Collectively, these observations argue that meta-experimental factors (such as the Hawthorn effect) were likely not primary factors in the present empirical findings.

\section{CONCLUSION}

It is critical to understand which visual processes can be altered through training and which cannot. The extent or generality of learning has broad implications for theories of vision and attention, applied questions of rehabilitation, and for how best to implement training protocols. To inform generalized learning, the current study implemented stroboscopic training. We observed improvements in motion detection and central attention, but not in sustained attention. These findings begin to narrow in on what processes can be trained and how, but future work is needed to fully understand the benefits of stroboscopic training. Interestingly, these results appear to be relatively robust in that similar effects were found after 2 days of training as well as after 10 days, and that effects were present for non-athletes and NCAA Division 1 varsity athletes alike. Open questions remain about the efficacy of training caused by differing levels or durations of exposure, the persistence of these training effects over time, and whether sleeprelated consolidation is required, but nevertheless, the current results provide an important step in understanding stroboscopic vision training, and generalized perceptual learning more broadly. Visual attention is a critical ability for many domains, and even a small increase can have profound effects; a small percent improvement in motion perception and focused attention may mean the world to an athlete engaged in a competitive sport, a sufferer of ADHD, or a soldier searching for an enemy combatant. The present 
results suggest that stroboscopic visual training may provide one specific means for improving visual cognitive abilities that could come to bare on these, and other, important activities.

\section{ACKNOWLEDGMENTS}

This research was funded by a grant to Stephen R. Mitroff from Nike SST, Nike Inc. Thanks to the Duke Athletic Trainers and

\section{REFERENCES}

Adcock, R. A., Thangavel, A., WhitfieldGabrieli, S., Knutson, B., and Gabrieli, J. D. (2006). Rewardmotivated learning: mesolimbic activation precedes memory formation. Neuron 50, 507-517.

Ahissar, M., and Hochstein, S. (2004). The reverse hierarchy theory of visual perceptual learning. Trends Cogn. Sci. (Regul. Ed.) 8, 457-464.

Ball, K., and Owsley, C. (1991). Identifying correlates of accident involvement for the older driver. Hum. Factors 33, 583-595.

Boot, W. R., Blakely, D. P., and Simons, D. J. (2011). Do action video games improve perception and cognition? Front. Psychol. 2:226. doi:10.3389/fpsyg.2011.00226

Crist, R. E., Kapadia, M. K., Westheimer, G., and Gilbert, C. D. (1997). Perceptual learning of spatial localization: specificity for orientation, position, and context. J. Neurophysiol. 78, 2889-2894.

Crundall, D., Underwood, G., and Chapman, P. (1999). Driving experience and the functional field of view. Perception 28, 1075-1087.

Desmurget, M., and Grafton, S. (2000). Forward modeling allows feedback control for fast reaching movements. Trends Cogn. Sci. (Regul. Ed.) 4, 423-431.

Donohue, S. E., Woldorff, M. G., and Mitroff, S. R. (2010). Video game players show more precise multisensory temporal processing abilities. Atten. Percept. Psychophys. 72, 1120-1129.

Dosher, B. A., and Lu, Z. L. (1999). Mechanisms of perceptual learning. Vision Res. 39, 3197-3221.

Fahle, M., and Poggio, T. (2002). Perceptual Learning. Cambridge: MIT Press.

Feng, J., Spence, I., and Pratt, J. (2007). Playing an action video game reduces gender differences in spatial cognition. Psychol. Sci. 18, 850-855.

Ferguson, C. J. (2007). The good, the bad and the ugly: a meta-analytic review of positive and negative effects of violent video games. Psychiatr. Q. 78, 309-316.

Fine, I., and Jacobs, R. A. (2002). Comparing perceptual learning tasks: a review. J. Vis. 2, 190-203.
Glover, S., and Dixon, P. (2004). Likelihood ratios: a simple and flexible statistic for empirical psychologists.

Goldstone, R. L. (1998). Perceptual learning. Annu. Rev. Psychol. 49, 585-612.

Green, C. S., and Bavelier, D. (2003). Action video game modifies visual 534-537.

Green, C. S., and Bavelier, D. (2006a). Effect of action video games on the spatial distribution of visuospatial attention. J. Exp. Psychol. Hum. Percept. Perform. 32, 1465-1478.

Green, C. S., and Bavelier, D. (2006b). Enumeration versus multiple object tracking: the case of action video game players. Cognition 101, 217-245.

Green, C. S., and Bavelier, D. (2008). Exercising your brain: a review of human brain plasticity and traininginduced learning. Psychol. Aging 23, 692-701.

Green, C. S., Li, R., and Bavelier, D. (2009). Perceptual learning during action video game playing. Top. Cogn. Sci. 2, 202-216.

Green, C. S., Pouget, A., and Bavelier, D. (2010). Improved probabilistic inference as a general learning mechanism with action video games. Curr. Biol. 20, 1573-1579.

Ho, Y. C., Cheung, M. C., and Chan, A. S. (2003). Music training improves verbal but not visual memory: crosssectional and longitudinal explorations in children. Neuropsychology 17, 439-450.

Kida, N., Oda, S., and Matsumura, M. (2005). Intensive baseball practice improves the go/nogo reaction time, but not the simple reaction time. Brain Res. Cogn. Brain Res. 22, 257-264. Michalopoulou, M., and Derri, V. (1998). Differences in several perceptual abilities between experts and novices in basketball, volleyball and water-polo. Percept. Mot. Skills 86, 899-912.

Koopman, S., Tsoi, L., and Wilmer, J. B. (2011). Keep your eye on the ball: watching and playing sports linked to smooth pursuit precision. J. Vis. $11,527$. Psychon. Bull. Rev. 11, 791-806. selective attention. Nature 423 ,

Kioumourtzoglou, E., Kourtessis, T.,

Coaches, the Duke Club Sports teams, all of the participants, as well as Stephen Adamo, Tym Blanchard, Christina Bogetich, Kait Clark, Ben Crisp, Chris Dall, Elise Darling, Sarah Donohue, Matt Forester, Katya Kabotyanski, Sylvia Nantier, Mike Schallmo, Rawan Shahin, Noha Sherif, and Ari Stern. Thanks to Tony Norcia and George Alvarez for providing code that the motion coherence and multiple-object tracking tasks, respectively, were based on.

Kraljic, T., and Samuel, A. G. (2006). Generalization in perceptual learning for speech. Psychon. Bull. Rev. 13, 262-268.

Li, R., Polat, U., Makous, W., and Bavelier, D. (2009). Enhancing the contrast sensitivity function through action video game training. Nat. Neurosci. 12, 549-551.

Li, R. W., Levi, D. M., and Klein, S. A. (2004). Perceptual learning improves efficiency by re-tuning the decision "template" for position discrimination. Nat. Neurosci. 7 , 178-183.

Lied, T. R., and Karzandjian, V. A. (1998). A Hawthorne strategy: implications for performance measurement and improvement. Clin. Perform. Qual. Health Care 6, 201-204.

Lum, J., Enns, J. T., and Pratt, J. (2002). Visual orienting in college athletes: explorations of athlete type and gender. Res. Q. Exerc. Sport 73, 156-167.

Mahncke, H. W., Connor, B. B., Appelman, J., Ahsanuddin, O. N., Hardy, J. L., Wood, R. A., Joyce, N. M., Boniske, T., Atkins, S. M., and Merzenich, M. M. (2006). Memory enhancement in healthy older adults using a brain plasticity-based training program: a randomized, controlled study. Proc. Natl. Acad. Sci. U.S.A. 103, 12523-12528.

Mann, D. T., Williams, A. M., Ward, P., and Janelle, C. M. (2007). Perceptual-cognitive expertise in sport: a meta-analysis. J. Sport Exerc. Psychol. 29, 457-478.

Marshall, L., and Born, J. (2007). The contribution of sleep to hippocampus-dependent memory consolidation. Trends Cogn. Sci. (Regul. Ed.) 11, 442-450.

Memmert, D., Simons, D. J., and Grimme, T. (2009). The relationship between visual attention and expertise in sports. Psychol. Sport. Exerc. 10, 146-151.

Myers, R. S., Ball, K. K., Kalina, T. D., Roth, D. L., and Goode, K. T. (2000). Relation of useful field of view and other screening tests to onroad driving performance. Percept. Mot. Skills 91, 279-290.

Proteau, L., and Cournoyer, J. (1990). Vision of the stylus in a manual aiming task: the effects of practice. Q. J. Exp. Psychol. A. 42, 811-828.

Proteau, L., Marteniuk, R. G., and Levesque, L. (1992). A sensorimotor basis for motor learning: evidence indicating specificity of practice. Q. J. Exp. Psychol. A. 44, 557-575.

Pylyshyn, Z. W., and Storm, R. W. (1988). Tracking multiple independent targets: evidence for a parallel tracking mechanism. Spat. Vis. 3, 179-197.

Raftery, A. E. (1995). "Bayesian model selection in social research," in Sociological Methodology 1995, ed. P.V Marsden (Cambridge, MA: Blackwell), 111-196.

Rauscher, F. H., Shaw, G. L., Levine, L. J., Wright, E. L., Dennis, W. R., and Newcomb, R. L. (1997). Music training causes long-term enhancement of preschool children's spatialtemporal reasoning. Neurol. Res. 19, 2-8.

Reschke, M. F., Krnavek, J. M., Somers, J. T., Ford, G., Hwang, E. J., Leigh, R. J., and Estrada, A. (2007). "Stroboscopic vision as a treatment for retinal slip induced motion sickness," in Proceedings of the First International Symposium on Visually Induced Motion Sickness, Fatigue, and Photosensitive Epileptic Seizures (VIMS2007) 1, Hong Kong, 51-58.

Reschke, M. F., Somers, J. T., and Ford, G. (2006). Stroboscopic vision as a treatment for motion sickness: strobe lighting vs. shutter glasses. Aviat. Space Environ. Med. 77, 2-7.

Richards, E., Bennett, P. J., and Sekuler, A. B. (2006). Age related differences in learning with the useful field of view. Vision Res. 46, 4217-4231.

Roenker, D. L., Cissell, G. M., Ball, K. K., Wadley, V. G., and Edwards, J. D. (2003). Speed-of-processing and driving simulator training result in improved driving performance. Hum. Factors 45, 218-233.

Savelsbergh, G. J., Williams, A. M., Van Der Kamp, J., and Ward, P. (2002). Visual search, anticipation and expertise in soccer goalkeepers. J. Sports Sci. 20, 279-287.

Schellenberg, E. G. (2004). Music lessons enhance IQ. Psychol. Sci. 15, 511-514. 
Sekuler, A. B., Bennett, P. J., and Mamelak, M. (2000). Effects of aging on the useful field of view. Exp. Aging Res. 26, 103-120.

Simon, H., and Chase, W. (1973). Skill in chess. Am. Sci. 61, 394-403.

Smith, G. E., Housen, P., Yaffe, K., Ruff, R., Kennison, R. F., Mahncke, H. W., and Zelinski, E. M. (2009). A cognitive training program based on principles of brain plasticity: results from the improvement in memory with plasticity-based adaptive cognitive training (IMPACT) study. J. Am. Geriatr. Soc. 57, 594-603.

Smith, T. Q. (2011). Sports Conditioning and Performance. Masters of Science in Sports Conditioning and Performance. Southern Utah University, Cedar City, UT.
Snowden, R. J., and Kavanagh, E. (2006). Motion perception in the ageing visual system: minimum motion, motion coherence, and speed discrimination thresholds. Perception 35, 9-24.

Tsimhoni, O., Yoo, H., and Green, P. (1999). Effects of Visual Demand Andin-Vehicle Task Complexity on Driving and Task Performance as Assessed by Visual Occlusion. The University of Michigan Transportation Research Institute (UMTRI); Mitsubishi Motors Corporation, Ann Arbor, MI.

Tsoi, L., Koopman, S., and Wilmer, J. B. (2011). Video-game training improves smooth pursuit precision. J. Vis. 11, 527.
Tsotsos, L. E., Roggeveen, A. B., Sekuler, A. B., Vrkljan, B. H., and Bennett, P. J. (2010). The effects of practice in a useful field of view task on driving performance. J. Vis. 10, 152.

Van Santen, J. P., and Sperling, G. (1985). Elaborated Reichardt detectors. J. Opt. Soc. Am. A 2, 300-321.

Wagenmakers, E. J. (2007). A practical solution to the pervasive problems of p values. Psychon. Bull. Rev. 14 779-804.

Conflict of Interest Statement: The authors declare that the research was conducted in the absence of any commercial or financial relationships that could be construed as a potential conflict of interest.
Received: 20 August 2011; accepted: 03 October 2011; published online: 28 October 2011.

Citation: Appelbaum LG, Schroeder JE, Cain MS and Mitroff SR (2011) Improved visual cognition through stroboscopic training. Front. Psychology 2:276. doi: 10.3389/fpsyg.2011.00276

This article was submitted to Frontiers in Perception Science, a specialty of Frontiers in Psychology.

Copyright (c) 2011 Appelbaum, Schroeder, Cain and Mitroff. This is an open-access article subject to a nonexclusive license between the authors and Frontiers Media SA, which permits use, distribution and reproduction in other forums, provided the original authors and source are credited and other Frontiers conditions are complied with. 